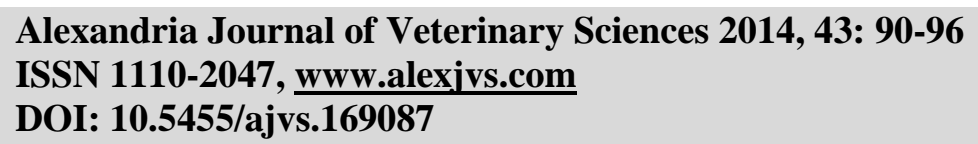

\title{
Detection of Circulating Immunoglobulins in Serum of Sheep Naturally Infected with Chlamydia Trachomatis and Listeria Monocytogenes in Two Egyptian Provinces.
}

\begin{abstract}
Shrief A. Zidan'; Reda Tarabees ${ }^{2}$ and Hamada Dahy ${ }^{3}$
1 Department of Animal Hygiene and Zoonoses, Faculty of Veterinary Medicine, Sadat City University, Al Menofia, Egypt.

2 Departememt of Bacteriology, Mycology, and immunology, Faculty of Veterinary Medicine, Sadat City University, Al Menofia, Egypt.

3 Department of Husbandry \& Animal wealth development Faculty of Veterinary Medicine, Sadat City University, Al Menofia, Egypt.

\section{Key words ABSTRACT:}

C. trachomatis, L. monocytogenes, ELISA

Chlamydia trachomatis and Listeria monocytogenes are important reproductive tract pathogens in a wide variety of animals including sheep. In humans, chronic or repeated infection of the female genital tract with Chlamydia trachomatis has been identified as a significant factor in the development of occlusive infertility or increased risk of ectopic pregnancy. The aim of the current study is to detect circulating immunoglobulins in serum of sheep naturally infected with Chlamydia trachomatis and Listeria monocytogenes in two Egyptian provinces. A total of 82 serum samples from 4 sheep flocks in two Egyptian provinces were examined with enzyme-linked immunosorbent assay (ELISA) for the detection of antibodies of Chlamydia trachomatis and Listeria monocytogenes. The results of ELISA revealed the detection of antibodies against the two pathogens at a very high percentage $(98.78 \%$ and $100 \%$ for Chlamydia trachomatis and Listeria monocytogenes, respectively) despite of absence of elevated abortion rates in the examined sheep flocks. It could be concluded that chlamydial and listerial infections occur frequently in Egyptian sheep flocks, even in the absence of elevated abortion rates and there is possible risk to pregnant women in rural areas where chlamydial and listerial infections in farm animals are widespread. Also, ewes that experienced Chlamydia trachomatis or Listeria monocytogenes induced abortion provide a unique opportunity to study the host: parasite dynamic as it relates to persistent infection. This natural model of persistent infection may, in some ways, be superior to more contrived models in which the chlamydial or listerial isolate is not a normal reproductive pathogen of the study animal. Thus, the study of persistent Chlamydial or Listerial infection in sheep may be used for the benefit of both human and veterinary medicine.
\end{abstract}

Corresponding Author: email: shrifzidan@yahoo.com

\section{INTRODUCTION}

The Chlamydiae are obligate intracellular parasites of eukaryotic cell found in the leucocytes and consist of minute particles. Chlamydiae are non-motile, coccoid, ranging from 0.2 to $1.5 \mathrm{um}$. Chlamydia trachomatis is composed of two biovars; the lymphogranuloma venereum agents and the ocular genital serotype, which may be distinguished by well-described seroprevalent with distinctive antigens in their outer membrane proteins. On the other hand, Listeria monocytogenes is an emerging bacterial foodborne pathogen responsible for listeriosis, an illness characterized by meningitis, encephalitis, and septicaemia. Less commonly, infection can result in cutaneous lesions and flu- like symptoms (Okoror et al. 2007 and Wesely, 1999). Chlamydiae and L. monocytogenes are important reproductive tract pathogens in a wide variety of animals. In humans, chronic or repeated infection of the female genital tract with C. trachomatis has been identified as a significant factor in the development of occlusive infertility or increased risk of ectopic pregnancy. Moreover, L. monocytogenes can cause bacteraemia, and stillbirth or premature birth of the fetus in pregnant women (Baud et al. 2008). The spectrum of reproductive disease recognized in sheep to be caused by Chlamydia or L. monocytogenes has been primarily restricted to pregnant animals because the 
organism was clearly identified as a major cause of infectious abortion. However, following pregnancy failure, a chronic chlamydial infection can become established in the reproductive tracts of experimentally infected ewes (Papp and Shewen, 1997).

Persistent infection of the ewe's reproductive tract may eventually result in pathology, similar to that observed in women infected with $\mathrm{C}$. trachomatis, thus decreasing the breeding life of affected ewes. Furthermore, ewes that experienced Chalmydia induced abortion provide a unique opportunity to study the host: parasite dynamic as it relates to persistent infection. This natural model of persistent infection may, in some ways, be superior to more contrived models in which the chlamydial isolate is not a normal reproductive pathogen of the study animal. Thus, the study of persistent chlamydial infection in sheep may be used for the benefit of both human and veterinary medicine (Papp and Shewen, 1997).

Listeria infection during pregnancy can cause miscarriage or premature birth. If the child is infected in the woman or during delivery this may lead to septicaemia and meningitis, which has a high fatality rate. There can also be longterm effects in many organs, including the airways, eyes and nervous system (Roberts and Wiedemann, 2003).

The fact that there are scarcity of data regarding the relations between $\mathrm{C}$. trachomatis, $\mathrm{L}$. monocytogenes and infertility cases in Egypt. Therefore, this study was aimed in the main to elucidate the seroprevalence of $\mathrm{C}$. trachomatis and L. monocytogenes infection among sheep, Moreover, C. trachomatis and L. monocytogenes are potentially zoonotic agents raise questions about the significance of this reservoir for animal and human health and underline the necessity for regular monitoring (Lenzko et al. 2011).

\section{MATERIALS AND METHODS:}

\subsection{Collection of blood Samples:}

A total of 82 blood samples were collected from 4 sheep flocks in two Egyptian provinces
(Menofia and Kafr El-Shiekh) throughout the period extended from September 2012 to March 2013. Each animal was identified according to age, locality, time of sampling and presence or absence of previous history of abortion. Samples were collected from the selected animal, left tightly closed in the refrigerator overnight at 4 ${ }^{\circ} \mathrm{C}$, and then centrifuged at $1500 \mathrm{rpm}$ for 15 minutes. Sera were obtained by using sterile Pasteur pipettes, labeled and stored at $-20{ }^{\circ} \mathrm{C}$ until examined for presence of $\mathrm{C}$. trachomatis and L. monocytogenes antibodies by ELISA.

\subsection{Enzyme Linked Immunosorbent assay (ELISA) for the detection of Chlamydia trachomatis in ovine's serum samples. (Catalogue No. 201-07-0049 SunRed®).} (Anusz et al., 1990).

It was used for in vitro diagnosis of $C$. trachomatis. It was an enzyme immunoassay for the determination of $C$. trachomatis in ovine serum, blood plasma and other related fluids and performed according to the instructions of the manufacturer company.

\section{Assay procedure:}

\section{Preparing reagents, samples and standards:}

\section{Standard solution:}

- The test supplies one original standard reagent $50 \mu \mathrm{l}$ which can be diluted according to the instruction manual

- The quantity of the plates depends on the quantities of to-be-tested samples and standards.

- It is suggested to duplicate each standard and blank well.

\section{Inject samples:}

- Blank well: do not add samples and CTantibody labeled with biotin, streptavidin-HRP, only chromagen solution A and B and stop solution are allowed other preparation are the same.

- Standard well: add standard $50 \mu \mathrm{l}$, streptavidinHRP $50 \mu \mathrm{l}$ (since the standard already has combined biotin antibody, it is not necessary to add the antibody).

- To the test wells: add samples $40 \mu \mathrm{l}$ and then add both CT-antibody $10 \mu \mathrm{l}$ and streptavidin-HRP 50 
$\mu 1$. then seal the sealing membrane and gently shaking, incubated 60 minutes at 37c.

- Confection: dilute 30 times the 30x washing concentrate with distilled water as standby.

- Washing: remove the membrane carefully, and then drain the liquid, shake away the remaining water.

2- Add chromagen solution A $50 \mu \mathrm{l}$, then chromagen solution B $50 \mu$ to each well. Gently mixed Incubate again at $\left(37^{\circ} \mathrm{C}\right)$ in the dark for 10 minutes followed by Adding $50 \mu \mathrm{l}$ stop reagent then carry out the photometric measurement at $450 \mathrm{~nm}$.

3- According to standards concentration and the corresponding OD values, calculate out the standard curve linear regression equation, and then apply the OD values of the sample on the regression equation to calculate the corresponding sample concentration or by using software programs.

\section{Sensitivity, assay range:}

Sensitivity: $18.553 \mathrm{ng} / \mathrm{L}$ (the sensitivity of the assay was defined as the lowest protein concentration that could be differentiated from zero. It is determined by sub-tracking two standards deviations to the mean optical density value of twenty zero standard replicates and calculating the corresponding concentration.)

Assay range: $20 \mathrm{ng} / \mathrm{L}-7200 \mathrm{ng} / \mathrm{L}$.

2.3. Enzyme Linked Immunosorbent assay (ELISA) for the detection of Listeria monocytogenes in ovine's serum samples (Catalogue No. 201-12-2067 SunRed®):

(Anusz et al., 1990).

The test was used for in vitro diagnosis of $L$. monocytogenes. It was an enzyme immunoassay for the determination of L. monocytogenes in ovine serum, blood plasma and other related fluids and performed according to instructions of the manufacturer company.

\section{Assay procedure:}

\section{Preparing reagents, samples and standards:}

\section{Standard solution:}

2. The test supplies one original standard reagent $50 \mu \mathrm{l}$ which can be diluted according to the instruction manual.
3. The quantity of the plates depends on the quantities of to-be-tested samples and standards.

It is suggested to duplicate each standard and blank well.

\section{Inject samples:}

- Blank well: do not add samples and LM-antibody labeled with biotin, streptavidin-HRP, only chromagen solution A and B and stop solution are allowed other preparation are the same.

- Standard well: add standard $50 \mu \mathrm{l}$, streptavidinHRP $50 \mu \mathrm{l}$ (since the standard already has combined biotin antibody, it is not necessary to add the antibody).

- To be the test wells: add samples $40 \mu \mathrm{l}$ and then add both LM-antibody $10 \mu \mathrm{l}$ and streptavidinHRP $50 \mu$ l. then seal the sealing membrane and gently shaking, incubated 60 minutes at 37c.

5. Confection: dilute 30 times the $30 \mathrm{x}$ washing concentrate with distilled water as standby.

6. Washing: remove the membrane carefully, and then drain the liquid, shake away the remaining water.

7. Add chromagen solution A $50 \mu$, then chromagen solution B $50 \mu$ lo each well. Gently mixed Incubate again at $\left(37 \mathrm{C}^{\circ}\right)$ in the dark for 10 minutes followed by Adding $50 \mu \mathrm{l}$ stop reagent and finally, carry out the photometric measurement at $450 \mathrm{~nm}$.

8. According to standards concentration and the corresponding OD values, calculate out the standard curve linear regression equation, and then apply the OD values of the sample on the regression equation to calculate the corresponding sample concentration or by using software programs.

\section{Sensitivity, assay range:}

Sensitivity: $0.1 \mathrm{nnmol} / \mathrm{L}$ (the sensitivity of this assay was defined as the lowest protein concentration that could be differentiated from zero. It is determined by sub-tracing two standards' deviations to the mean optical density value of twenty zero standard replicates and calculating the corresponding concentration.)

Assay range: $0.1 \mathrm{nmol} / \mathrm{L}-27 \mathrm{nmol} / \mathrm{L}$. 


\section{RESULTS}

Table (1): The results of indirect enzyme linked immunosorbant assay (ELISA) for detection of circulating immunoglobulin in serum of sheep naturally infected with C. trachomatis in two Egyptian provinces

\begin{tabular}{ccccccc}
\hline \hline Province & $\begin{array}{c}\text { No. of examined } \\
\text { sheep }\end{array}$ & $+\mathrm{ve}$ & $\%$ & $-\mathrm{ve}$ & $\%$ & $\begin{array}{c}\text { mean absorbance of } \\
\text { positive sera } \pm \text { SD } \\
(\mathrm{ng} / \mathrm{L})\end{array}$ \\
\hline Menofia & 40 & 39 & $97.5 \%$ & 1 & $2.5 \%$ & $1889.21 \pm 678.7$ \\
Kafr ELshiekh & 42 & 42 & 100 & 0 & 0 & $1767.81 \pm 804.13$ \\
\hline Total & 82 & 81 & 98.78 & 1 & 1.22 & \\
\hline \hline
\end{tabular}

Determined at $450 \mathrm{~nm}$ wave length and cutoff level corresponding to $18.553 \mathrm{ng} / \mathrm{L}$ (the lowest protein concentration that could be differentiated from zero).

\section{Standard Curve for Chlamidia Trachomites}

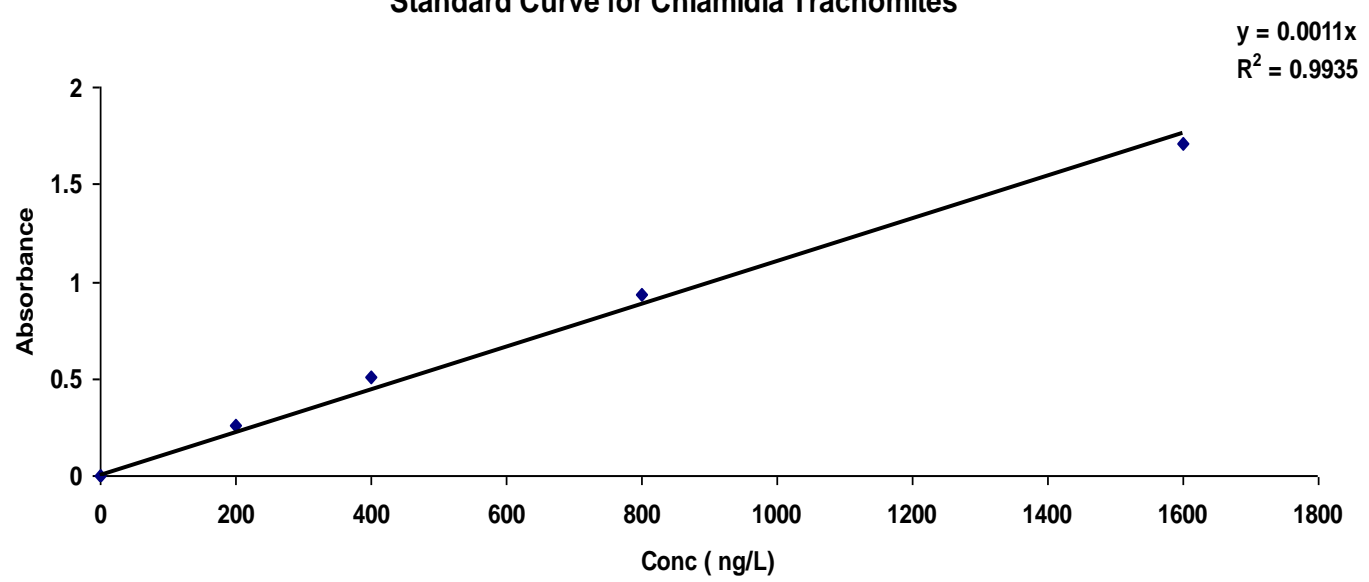

Fig (1): Graph illustrates the standard curve obtained from ovine $C$. trachomatis ELISA test. The concentration of the protein $(\mathrm{ngl} / \mathrm{L})$ in each sample was obtained the equation showed on the graph. The equation corresponding to a linear fit of the absorbance.

Table (2): The results of indirect enzyme linked immunosorbant assay (ELISA) for detection of circulating immunoglobulins in serum of sheep naturally infected with L. monocytogens in two Egyptian provinces.

\begin{tabular}{ccccccc}
\hline \hline Province & $\begin{array}{c}\text { No. of examined } \\
\text { sheep }\end{array}$ & Positive & $\%$ & Negative & $\%$ & $\begin{array}{c}\text { mean absorbance of positive } \\
\text { sera } \pm \text { SD } \\
(\mathrm{nmol} / \mathrm{L})\end{array}$ \\
\hline Menofia & 40 & 40 & 100 & 0 & 0 & $7.61 \pm 2.67$ \\
Kafr ELshiekh & 42 & 42 & 100 & 0 & 0 & $5.71 \pm 2.51$ \\
\hline Total & 82 & 82 & 100 & 0 & 0 & \\
\hline \hline
\end{tabular}

Determined at $450 \mathrm{~nm}$ wave length and cutoff level $0.1 \mathrm{nmol} / \mathrm{L}$ (the lowest protein concentration that could be differentiated from zero). 


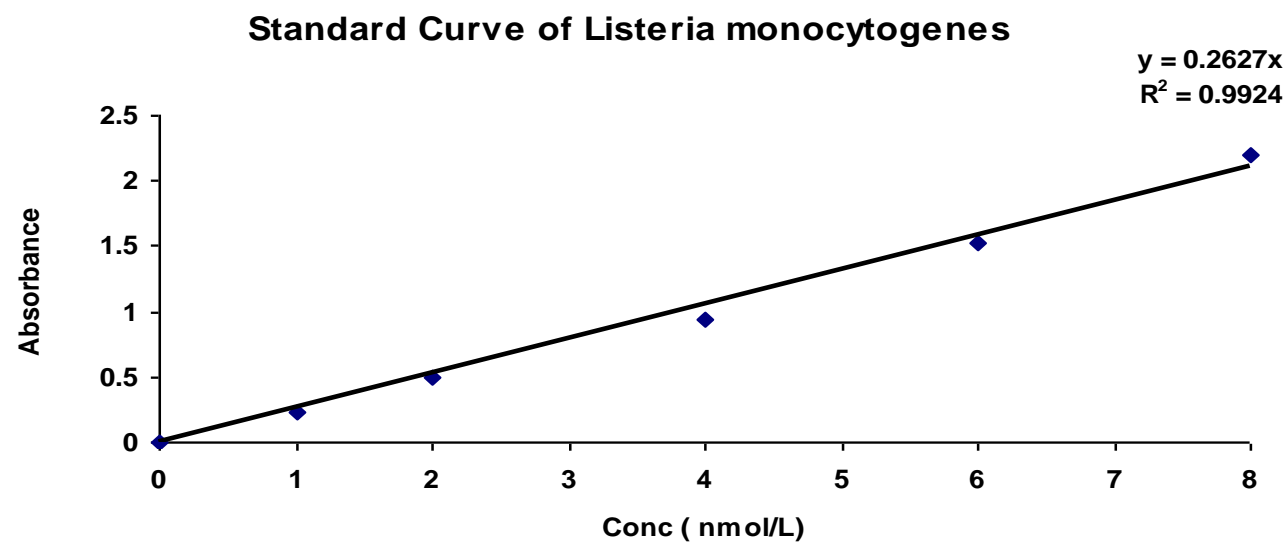

Fig (2): Graph illustrates the standard curve obtained from listeria monocytogenes ELISA test. The concentration of the protein $(\mathrm{nmol} / \mathrm{L})$ in each sample was obtained the equation showed on the graph. The equation corresponding to a linear fit of the absorbance.

\section{DISCUSSION}

C. trachomatis and L. monocytogenes are two important zoonotic pathogens (Low and Donachie, 1997 and Baud et al. 2008). $C$. trachomatis causes about $40 \%$ to approximately $50 \%$ non gonococcal urethritis in men, epididymitis, mucopurulent cervicitis, proctitis, urethritis, endometritis, salpingitis, endemic trachoma, inclusion conjunctivitis, newborn pneumonia, later post-partum endometritis, pelvic inflammatory diseases in women and cervical pains. It can also cause post gonococcal urethritis. The pelvic inflammatory diseases often result in infertility and ectopic pregnancy.

The epidemiological situation of ovine chlamydial and listerial infections in continental Africa, especially Egypt is poorly characterized. Using the Menofia and Kafr Elshiekh as a model example, the chlamydial and listerial seroprevalence was estimated in four randomly selected sheep flocks with an average abortion rate lower than $5 \%$.

A total of 82 samples from 4 flocks in two Egyptian governorates (El Menofia and Kafr ElSheikh) were examined by ELISA for detection of antibodies of $C$. trachomatis and Listeria monocytogenes. The recorded result in Tables 1 and 2 revealed that $98.78 \%$ and $100 \%$ of the tested sheep samples were serologically positive with ongoing infection for $C$. trachomatis and $L$. monocytogenes, respectively although the elevated abortion rates was absent in the examined sheep flocks. Moreover, the results were nearly similar in the two governorates for $C$. trachomatis and $L$. monocytogenes (Tables 1 and 2). C. trachomatis was detected in 39 out of $40(97.5 \%)$ samples in Menofia governorate with mean absorbance of positive sera of $1889.21 \pm 678.7$ while it was detected in 42 out $42(100 \%)$ samples in Kafr ElShiekh with mean absorbance of positive sera of 1767.81 \pm 804.13 . On the other hand, $L$. monocytogenes was detected in 40 out of 40 (100\%) samples in Menofia governorate with mean absorbance of positive sera of 7.61 \pm 2.67 while it was detected in 42 out $42(100 \%)$ samples in Kafr ElShiekh with mean absorbance of positive sera of 5.71 \pm 2.51 . Among several authors who studied the infectious causes of abortion in animals. Lenzko et al. (2011) reported that $94 \%$ of the tested sheep flocks were serologically positive with chalamydial infection in Germany. Moreover, Husu, (1990) and stated that L. monocytogenes was a ubiquitous organism, and regular exposure of animals and humans to this microorganism was very common. Furthermore, many healthy individuals were intestinal carriers (2-6\%) and anti- $L$. monocytogenes serum antibody prevalence as high 
as $53 \%$ have been reported in humans. Carriage rate for animals was similar to that of humans, with some differences depending on the species and a little higher rate during indoor season, as compared to animals on pasture (Iida et al. 1991).

On the other hand, Yildiz et al. (2009) determined seroprevalence of Neospora caninum in dairy cattle with higher abortion rates and coexistence of $N$. caninum with L. monocytogenes antibodies and indicated that $N$. caninum seroprevalence was $10.77 \% \quad(60 / 557)$ while the co-existence rate of $N$. caninum seropositivity with L. monocytogenes was $42.85 \%$ (162/378). Moreover, AbdEl-Malek, et al ( 2010) examined 28 stool cultures from hospitalized children with diarrheal disease in Assiut Univ. hospital Egypt, 2 (7.14\%) were found positive for Listeria monocytogenes using polymerase chain reaction (PCR).

Reproductive disease of sheep caused by Chlamydia spp. and L. monocytogenes has been primarily restricted to pregnant ewes because the organism was clearly identified as a major cause of infectious abortion. However, following pregnancy failure, a chronic chlamydial infection can become established in the reproductive tracts of experimentally infected animals. Persistent infection of the ewe's reproductive tract may eventually result in pathological lesions, similar to that observed in women infected with $C$. trachomatis, thus decreasing the breeding life of affected ewes (Papp and Shewen, 1997).

Infection within flocks of sheep in which chlamydial abortion has occurred may be maintained between lambing seasons by enteric spread from infected ewes to lambs, and subsequently between lambs, to give a persistent but mainly subclinical gut infection. Placental infection, when the ewes mature and become pregnant, might arise by the selection of variants with affinity for the placenta from the pre-existing enteric infection (Johnson et al. 1985).

The public must be aware of the possible risk to pregnant women in rural areas where chlamydial and listerial infections of farm animals are widespread. Women who are, or who may be, pregnant are potentially at risk of acquiring certain infectious diseases from pregnant livestock including sheep. Also, it is important to note that these risks are not only confined to the spring (when the majority of lambs are born), nor are the risks only associated with sheep. Cows and goats that have recently given birth can also carry similar infections (Low and Donachie, 1997 and Baud et al. 2008).

Infection with certain organisms can cause abortion in pregnant sheep, and if a pregnant woman becomes infected with these organisms they may harm her unborn child. The most important organisms are $C$. trachomatis which causes abortion of ewes, Toxoplasma gondii, $L$. monocytogenes and also Q fever which, although less likely to cause harm to the unborn child, may cause infection in the mother (Baud et al. 2008).

Such women should be advised against close contact with animals during their pregnancy and, especially, helping with sheep during the lambing season. If clinically similar cases occur medical attendants should recognize that confirmatory laboratory tests are available, including tests for ELISA to Chlamydia and listeria and, in cases of abortion, the simple microscopic examination of smears of the placenta stained with Giemsa (Low and Donachie, 1997 and Baud et al. 2008).

Based on the obtained results in the current study, it was clear that chlamydial and listerial infections occur frequently in Egyptian sheep flocks, even in the absence of elevated abortion rates.

The fact that $C$. trachomatis or $L$. monocytogenes are potentially zoonotic pathogens, raise questions about the significance of this reservoir for animal and human health and underline the necessity for regular monitoring. Further studies are needed to identify precisely the possible role of $C$. trachomatis or $L$. monocytogenes infections in woman abortion in Egypt.

\section{ACKNOWLEDGEMENT}

This project was supported financially by the Science and Technology Development Fund (STDF), Egypt. Grant No. 736. 


\section{REFERENCES}

AbdEl-Malek,A.M., Hassan Ali,S.F., Moemen,R.H., Mohamed,A. and Elsayh,K.I. 2010.Occurrence of Listeria species in meat, chicken products and human stools in Assiut city, Egypt with PCR use for rapid identification of Listeria monocytogenes. Vet World. ; 3(8): 353-359.

Anusz, K. Z., Manson, P. H., Riggs, M. W. and Perryman, L. E. 1990: Detection of Cryotosporidium parvum oocysts in bovine faeces by monoclonal antibody capture enzyme linked immunosorbant assay. J. Clin. Microbiol., 28: 2770-2774.

Baud, D., Regan, L. Greub, G. 2008. Emerging role of Chlamydia and Chlamydia-like organisms in adverse pregnancy outcomes. Curr. Opin. Infect. 21(1): 70-76.

Husu, J. R. 1990. Epidemiological studies on the occurrence of Listeria monocytogenes in the feces of dairy cattle. J. Vet. Med. (37): 276-282.

Iida, T., Kanzaki, M., Maryuma, T., Inoue S. Kaneuchi, C. 1991. Prevalence of Listeria monocytogenes in intestinal contents of healthy animals in Japan. J. Vet. Med. Sci., (53): 873-875.

Johnson, F. W. A., Mathson, B. A., William, H., lanig, A. G., Jandial, D. G., Halliday, D., Hobson, S. Y., Wong, S., Kathrene, M., Moffat, A. J., Postlethwaite, R. 1985. Abortion due to infection with Chlamydia psittaci in sheep farmer's wife. British med. J. (290): 23-31
Lenzko, H., Moog, U., Henning, K., Lederbach, R., Diller, R., Menge, C., Sachse, K., Sprague, L. D. 2011. High frequency of chlamydial co-infections in clinically healthy sheep flocks. BMC Vet Res. (10): -7-29.

Low, J. C., Donachie, W. 1997. A review of Listeria monocytogenes and listeriosis. Vet. J., (153): 9-29.

Okoror, L. E., Agbonlahor, D. E., Esumeh, F. I. Umolu, P. I. 2007. Prevalence of chlamydia in patients attending gynecological clinics in South Eastern Nigeria. Afr. Health Sci. 7 (1): 18-24.

Papp, J. R. Shewen, P. E. 1997. Chlamydia psittaci infection in sheep: a paradigm for human reproductive tract infection. J. Reprod. Immunol. 34 (3): 185-202.

Roberts, A. J., Wiedemann, M. 2003. Pathogen, host and environmental factors contributing to the pathogenesis of listeriosis. Cell. Mol. Life Sci., (60): 904-918.

Wesiely, G. N. 1999. Listeriosis in animals. In: Listeria, Listeriosis, and Food Safety, Ryser E. \& Marth E.,eds. Marcel Dekker, New York, NY, USA, 39-73.

Yildiz, K., Kul O., Babur, C., Kilic, S., Gazyagci, A. N., Celebi, B. Gurcan, I. S. 2009. Seroprevalence of Neospora caninum in dairy cattle ranches with high abortion rate: special emphasis to serologic co-existence with Toxoplasma gondii, Brucella abortus and Listeria monocytogenes. Vet Parasitol. 14, 164 (2-4): 306-10. 